\title{
The correlation structure of spatial autoregressions
}

Article

Accepted Version

Martellosio, F. (2012) The correlation structure of spatial autoregressions. Econometric Theory, 28 (6). pp. 1373-1391. ISSN 1469-4360 doi:

https://doi.org/10.1017/S0266466612000175 Available at https://centaur.reading.ac.uk/24472/

It is advisable to refer to the publisher's version if you intend to cite from the work. See Guidance on citing.

To link to this article DOI: http://dx.doi.org/10.1017/S0266466612000175

Publisher: Cambridge University Press

All outputs in CentAUR are protected by Intellectual Property Rights law, including copyright law. Copyright and IPR is retained by the creators or other copyright holders. Terms and conditions for use of this material are defined in the End User Agreement.

\section{www.reading.ac.uk/centaur}

\section{CentAUR}

Central Archive at the University of Reading

Reading's research outputs online 


\title{
The Correlation Structure of Spatial Autoregressions
}

\author{
Federico Martellosio \\ University of Reading \\ October 19, 2011
}

Acknowledgements: I am grateful to the co-editor Guido Kuersteiner, four anonymous referees, Grant Hillier, Paolo Paruolo and Tony Smith for helpful comments.

Mailing address: Department of Economics, University of Reading, Whiteknights, Reading RG6 6AA, UK. Tel: +44 (0) 1183786033.

E-mail: f.martellosio@reading.ac.uk 
Running head: The Correlation Structure of SAR Models

Corresponding author: Federico Martellosio

\begin{abstract}
This paper investigates how the correlations implied by a first-order simultaneous autoregressive $(\mathrm{SAR}(1))$ process are affected by the weights matrix and the autocorrelation parameter. A graph theoretic representation of the covariances in terms of walks connecting the spatial units helps to clarify a number of correlation properties of the processes. In particular, we study some implications of row-standardizing the weights matrix, the dependence of the correlations on graph distance, and the behavior of the correlations at the extremes of the parameter space. Throughout the analysis differences between directed and undirected networks are emphasized. The graph theoretic representation also clarifies why it is difficult to relate properties of $\boldsymbol{W}$ to correlation properties of SAR(1) models defined on irregular lattices.
\end{abstract}




\section{Introduction}

First-order simultaneous autoregressive $(\operatorname{SAR}(1))$ processes are widely used to model interaction over a geographic, economic, or social space (e.g., Cliff and Ord, 1981; Anselin, 1988; LeSage and Pace, 2009). Such processes postulate a simple relationship between each of its two main components - the weights matrix $\boldsymbol{W}$ and the correlation parameter $\rho$-and the inverse of their variance matrix. Due to the matrix inversion, however, the way in which $\boldsymbol{W}$ and $\rho$ affect the correlations implied by the model may be difficult to understand. Wall (2004) discusses some unexpected correlation properties of SAR(1) models defined on irregularly spaced lattices, and concludes that the models exhibit counterintuitive behavior. Apart from that paper, there has not been much work attempting to clarify the correlation properties of SAR(1) models on irregular lattices. On the other hand, the correlation structure of SAR(1) models is well-understood when the observational units form a regular lattice (e.g., Whittle, 1954; Besag, 1972; Bartlett, 1975). In economics, regular lattices are the norm in the time-series case, but are extremely rare in higher dimensional spaces.

The present paper is a theoretical investigation of the correlation structure of $\operatorname{SAR}(1)$ processes defined on general configurations of observational units. We provide a representation of the covariances between two variables observed at two spatial units as the sum of the contributions coming from (a particular type of) walks connecting the two units. The representation helps to understand several properties of SAR(1) models, and it clarifies the role of $\rho$ in shaping the correlation structure of the models. The parameter $\rho$ controls the relative importance of contributions coming from walks of different length. When $|\rho|$ is small the correlation structure is largely determined by short walks, but, as $|\rho|$ increases, the importance of longer walks increases. This implies that SAR(1) models may exhibit significantly different correlation properties depending on the value of $\rho$. Since $\rho$ is unknown, and is estimated only after $\boldsymbol{W}$ has been fixed, it follows that in empirical applications it is difficult, or even impossible, to control the correlation properties of a $\operatorname{SAR}(1)$ model through the specification of $\boldsymbol{W}$.

The rest of the paper is organized as follows. SAR(1) processes are presented in Section 2. Section 3 introduces some graph theoretic terminology, which is then used in Section 4 to formulate the representation of the covariance structure of SAR(1) models. Section 5 analyzes the correlation properties of SAR(1) models. In particular, we study the effect of row-standardizing $\boldsymbol{W}$, the dependence of the correlations on distance, and the behavior of the correlations at the extremes of the parameter space. Throughout the analysis several consequences of the common practice of row-standardizing $\boldsymbol{W}$ are pointed out. Particular attention is paid to the case of directed networks, which is important in several economic applications. Section 6 concludes. Proofs are collected in the Appendix.

\section{Specification of the $\operatorname{SAR}(1)$ Model}

Consider a fixed and finite set of $n$ observational units. The units are labelled by the first $n$ integers in some arbitrary way, and the $i$-th unit has the random variable $y_{i}$ associated with 
it. Letting $\boldsymbol{y}=\left(y_{1}, \ldots, y_{n}\right)^{\prime}$ be a zero-mean random vector, a $\mathrm{SAR}(1)$ model is given by

$$
\boldsymbol{y}=\rho \boldsymbol{W} \boldsymbol{y}+\boldsymbol{\varepsilon},
$$

where $\rho$ is a real unknown parameter, $\boldsymbol{W}$ is an $n \times n$ weights matrix, $\varepsilon$ is a vector of errors such that $\mathrm{E}(\boldsymbol{\varepsilon})=\mathbf{0}$ and $\operatorname{var}(\varepsilon)=\sigma^{2} \boldsymbol{V}$, with $\sigma^{2}>0$ and $\boldsymbol{V}$ a known $n \times n$ diagonal (and positive definite) matrix. Because in this paper we are only interested in correlations, we do not need to consider extensions of $(1)$ to the case $\mathrm{E}(\boldsymbol{y}) \neq \mathbf{0}{ }^{1}$

The matrix $\boldsymbol{W}$ is non-stochastic and known. It is chosen to reflect a priori information on pairwise relations between the observational units (see, e.g., Bavaud, 1998, and Griffith and Lagona, 1998). For example, the entries $(\boldsymbol{W})_{i j}$ may be taken to be a certain function of some physical or economic distance between the $i$-th and the $j$-th observational units.

For the purpose of studying the correlation structure of SAR(1) models, we can set $\sigma^{2}=1$ and $\boldsymbol{V}=\boldsymbol{I}_{n}$ (the identity matrix of order $n$ ) without loss of generality. ${ }^{2}$ Accordingly, for any $\rho$ different from the reciprocal of the nonzero real eigenvalues of $\boldsymbol{W}$, model (1) implies the variance matrix

$$
\operatorname{var}(\boldsymbol{y})=\left[\left(\boldsymbol{I}_{n}-\rho \boldsymbol{W}^{\prime}\right)\left(\boldsymbol{I}_{n}-\rho \boldsymbol{W}\right)\right]^{-1}=: \boldsymbol{\Sigma}(\rho) .
$$

For convenience, throughout the paper we maintain the following two assumptions, which are usually satisfied in applications.

Assumption 2.1 For any $i, j=1, \ldots, n,(\boldsymbol{W})_{i j} \geq 0$.

Assumption 2.2 For any $i=1, \ldots, n,(\boldsymbol{W})_{i i}=0$.

Assumption 2.1 allows us to use results on nonnegative matrices (e.g., Horn and Johnson, 1985). Assumption 2.2 has consequences on the properties of the graph distance to be defined in the next section.

Let $\lambda_{\max }$ denote the spectral radius (i.e., the largest modulus of the eigenvalues) of $\boldsymbol{W}$. Since $\boldsymbol{W}$ is nonnegative, $\lambda_{\max }$ is an eigenvalue of $\boldsymbol{W}$ (Horn and Johnson, 1985, Theorem 8.3.1). Also, when $\boldsymbol{W}$ has at least one (real) negative eigenvalue, we denote the smallest negative eigenvalue by $\lambda_{\min }$. Then, the largest connected interval of values of $\rho$ around the origin where $\boldsymbol{\Sigma}(\rho)$ exists is $\left(\rho_{\min }, \rho_{\max }\right)$, with $\rho_{\min }:=\lambda_{\min }^{-1}$ if $\boldsymbol{W}$ has at least one negative eigenvalue, $\rho_{\min }:=-\infty$ otherwise, and with $\rho_{\max }:=\lambda_{\max }^{-1}$ if $\lambda_{\max }>0, \rho_{\max }:=\infty$ if

$\lambda_{\max }=0 .{ }^{3}$ In the literature, some authors take $\left(\rho_{\min }, \rho_{\max }\right)$ as the set of admissible values for $\rho$, whereas other authors choose its subset $\left(-\rho_{\max }, \rho_{\max }\right)$. In Section 4 we shall see that the latter choice has the advantage of guaranteeing a simple interpretation of the correlation structure of the model.

\section{Some Graph Theoretic Notions}

For our analysis, it is useful to consider the graph underlying $\boldsymbol{W}$, that is, the graph having as vertices the $n$ observational units, and as arcs the ordered pairs $(i, j)$ such that $(\boldsymbol{W})_{i j}>0$. For a general introduction to graph theory see, e.g., Harary (1969). 
In graph theory, a (directed) walk from a vertex $i_{0}$ to a vertex $i_{r}$ is an alternating sequence $\left(i_{0}, a_{1}, i_{1}, \ldots, a_{r}, i_{r}\right), r \geq 0$, of vertices and arcs in which each arc $a_{t}$ is $\left(i_{t-1}, i_{t}\right)$. For the purposes of this paper, we need to modify the definition of a walk as follows.

Definition 3.1 A SAR-walk from $i_{0}$ to $i_{r}$ is an alternating sequence $\left(i_{0}, a_{1}, i_{1}, \ldots, a_{r}, i_{r}\right)$ of vertices and arcs in which, for some $k=0, \ldots, r$, the first $k$ arcs $a_{t}$ are $\left(i_{t-1}, i_{t}\right)$ and the remaining $r-k$ arcs are $a_{t}=\left(i_{t}, i_{t-1}\right)$.

Note that a SAR-walk is a walk only for $k=r$. For $k<r$, the first $k$ steps of a $\mathrm{SAR}$-walk are in the direction of the sequence $\left(i_{0}, a_{1}, i_{1}, \ldots, a_{r}, i_{r}\right)$ and the remaining ones are in the opposite direction. A SAR-walk will generally be denoted using the notation $\left(i_{0} \rightarrow i_{1} \rightarrow \ldots \rightarrow i_{k} \leftarrow \ldots \leftarrow i_{r-1} \leftarrow i_{r}\right)$. The following example illustrates the difference between walks and SAR-walks.

Example 3.2 Figure 1 displays the graph underlying the weights matrix

$$
\boldsymbol{W}=\left[\begin{array}{lllll}
0 & 1 & 0 & 0 & 0 \\
1 & 0 & 1 & 0 & 0 \\
0 & 0 & 0 & 0 & 0 \\
0 & 0 & 1 & 0 & 1 \\
0 & 0 & 0 & 1 & 0
\end{array}\right]
$$

A line with an arrowhead represents an arc, and a line joining two vertices $i$ and $j$ without an arrowhead indicates that there is both an arc from $i$ to $j$ and an arc from $j$ to $i$. Observe that there are no walks from unit 2 to unit 4. However, there are SAR-walks joining 2 and 4: for instance $(2 \rightarrow 3 \leftarrow 4)$ is a SAR-walk of length 2 from 2 to 4 .

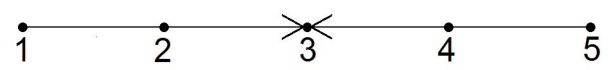

Figure 1: The graph underlying the weights matrix (3).

We shall see in the next section that the notion of a SAR-walk is naturally suggested by a SAR(1) model, and that all SAR-walks from a unit $i$ to a unit $j$, not only those that are walks, give a contribution to $\operatorname{corr}\left(y_{i}, y_{j}\right)$. Accordingly, in order for $\operatorname{corr}\left(y_{i}, y_{j}\right)$ to be nonzero it is not necessary that $i$ and $j$ are joined by a walk, but that they are joined by a SAR-walk. For a plot of $\operatorname{corr}\left(y_{2}, y_{4}\right)$ implied by a $\operatorname{SAR}(1)$ model with weights matrix (3) see Figure 4.

Next, we adapt a number of standard graph theoretic notions to our definition of a SAR-walk. We say that a graph is connected if there is a SAR-walk from $i$ to $j$, for all $i, j=1, \ldots, n$. The length of a SAR-walk is the number of arcs in it ( $r$ in the case of the SAR-walk in Definition 3.1). The trivial sequence $\left(i_{0}\right)$ is to be regarded as a SAR-walk of length 0 from $i_{0}$ to $i_{0}$.

The definition of a SAR-walk imposes the following distance on the set of observational units.

Definition 3.3 The distance $d(i, j)$ between any two units $i$ and $j$ is the length of a shortest $S A R$-walk from $i$ to $j$, if any. Otherwise, $d(i, j)=\infty$. 
It is immediately clear that: (a) $d(i, j)=0$ if and only if $i=j$; (b) $d(i, j)=d(j, i)$ (which is why we speak of the distance between $i$ and $j$, rather than from $i$ to $j$ ); (c) $d(i, j)=1$ if and only if $(\boldsymbol{W})_{i j}+(\boldsymbol{W})_{j i}>0$. It is also easily checked that, if the graph is connected, then $d(\cdot, \cdot)$ satisfies the triangle inequality, and hence is a metric.

SAR-walks and the induced distance $d(\cdot, \cdot)$ depend only on which entries of $\boldsymbol{W}$ are zero and which are nonzero. It is also convenient to introduce a measure of the importance of different SAR-walks, based on the magnitude of the nonzero entries of $\boldsymbol{W}$. To each $\operatorname{arc}(i, j)$ we assign the weight $(\boldsymbol{W})_{i j}$. Then, based on a standard graph theoretic notion (e.g., Godsil, 1993, p. 56), we can define the weight of a SAR-walk as follows.

Definition 3.4 The weight of a SAR-walk is the product of the weights of its arcs if the $S A R$-walk has positive length. Otherwise, it is 1 .

We shall also use the following terminology. If $(\boldsymbol{W})_{i j}>0, j$ is said to be a neighbor of $i$, which we indicate in symbols by $i \rightarrow j$ (or $j \leftarrow i$ ). When $i \rightarrow j$ and $j \rightarrow i$, we say that $i$ and $j$ are neighbors. A graph such that $(\boldsymbol{W})_{i j}=0$ if and only if $(\boldsymbol{W})_{j i}=0$, for all $i, j=1, \ldots, n$, is said to be undirected; otherwise, it is said to be directed.

\section{A Graph Theoretic Representation of the Covariances}

For $r=0,1, \ldots, \infty$, let

$$
\boldsymbol{Z}_{r}:=\sum_{k=0}^{r} \boldsymbol{W}^{k}\left(\boldsymbol{W}^{\prime}\right)^{r-k},
$$

where, as usual, $\boldsymbol{W}^{0}:=\boldsymbol{I}_{n}$. The entries of the matrices $\boldsymbol{Z}_{r}$ admit the following interpretation.

Lemma $4.1\left(\boldsymbol{Z}_{r}\right)_{i j}$ equals the total weight of the SAR-walks of length $r$ from $i$ to $j$.

The next lemma follows almost immediately.

Lemma $4.2\left(\boldsymbol{Z}_{r}\right)_{i j}=0$ if and only if $r<d(i, j)$.

The matrices $\boldsymbol{Z}_{r}$ play an important role in our analysis, because they appear in an expansion of $\operatorname{cov}\left(y_{i}, y_{j}\right)$ in powers of $\rho$. When $|\rho| \lambda_{\max }<1,\left(\boldsymbol{I}_{n}-\rho \boldsymbol{W}\right)^{-1}=\sum_{r=0}^{\infty}(\rho \boldsymbol{W})^{r}$, and hence $\boldsymbol{\Sigma}(\rho)=\sum_{r, s=0}^{\infty} \rho^{r+s} \boldsymbol{W}^{r}\left(\boldsymbol{W}^{\prime}\right)^{s}=\sum_{r=0}^{\infty} \rho^{r} \boldsymbol{Z}_{r}$. Thus, by Lemma 4.2, we have that, when $|\rho| \lambda_{\max }<1$, and for any $i, j=1, \ldots, n$,

$$
\operatorname{cov}\left(y_{i}, y_{j}\right)=\sum_{r=d(i, j)}^{\infty}\left(\boldsymbol{Z}_{r}\right)_{i j} \rho^{r} .
$$

Let now $\mathcal{K}_{i j}$ be the set of all SAR-walks from $i$ to $j$, and let len $(\omega)$ and wei $(\omega)$ denote the length and the weight of a SAR-walk $\omega$. In light of Lemma 4.1, expression (5) leads to the following graph theoretic representation of $\operatorname{cov}\left(y_{i}, y_{j}\right)$. 
Theorem 4.3 In a $S A R(1)$ model, when $|\rho| \lambda_{\max }<1$, and for any $i, j=1, \ldots, n$,

$$
\operatorname{cov}\left(y_{i}, y_{j}\right)=\sum_{\omega \in \mathcal{K}_{i j}} \operatorname{wei}(\omega) \rho^{\operatorname{len}(\omega)}
$$

Theorem 4.3 relates the covariance structure of a SAR(1) model to the walk structure of the graph underlying $\boldsymbol{W}$. More specifically, it asserts that $\operatorname{cov}\left(y_{i}, y_{j}\right)$ can be interpreted as the sum of the contributions of all SAR-walks from $i$ to $j$, the contribution of a SAR-walk $\omega$ being wei $(\omega) \rho^{\operatorname{len}(\omega)}$. Such an interpretation holds for $|\rho| \lambda_{\max }<1$, or, equivalently, for $\rho \in\left(-\rho_{\max }, \rho_{\max }\right)$.

Example 4.4 Consider the very simple weights matrix

$$
\boldsymbol{W}=\left[\begin{array}{ll}
0 & 1 \\
0 & 0
\end{array}\right] \text {. }
$$

The underlying graph consists of the vertices 1 and 2 , with an arc from 1 to 2 . There is only one SAR-walk from 1 to 2, with length and weight both equal to 1 . Hence, by Theorem 4.3, $\operatorname{cov}\left(y_{1}, y_{2}\right)=\rho$. This expression holds for any $\rho$, because $\lambda_{\max }=0$ for matrix (7). An alternative to the weights matrix (7) is

$$
\boldsymbol{W}=\left[\begin{array}{ll}
0 & 1 \\
1 & 0
\end{array}\right],
$$

which specifies symmetric interaction between units 1 and 2 , and has $\lambda_{\max }=1$. The extra arc from 2 to 1 implies that there are SAR-walks of any odd length contributing to $\operatorname{cov}\left(y_{1}, y_{2}\right)$. More specifically, for any odd $r$, there are $r+1$ SAR-walks of length $r$ and weight 1 from 1 to 2 (for $r=1$, they are: $(1 \rightarrow 2)$ and $(1 \leftarrow 2)$; for $r=3$, they are: $(1 \rightarrow 2 \rightarrow 1 \rightarrow 2)$, $(1 \rightarrow 2 \rightarrow 1 \leftarrow 2),(1 \rightarrow 2 \leftarrow 1 \leftarrow 2)$, and $(1 \leftarrow 2 \leftarrow 1 \leftarrow 2)$; and so on). Using Theorem 4.3 we then obtain that, for $|\rho|<1, \operatorname{cov}\left(y_{1}, y_{2}\right)=\sum_{r=0 ; r \text { odd }}^{\infty}(r+1) \rho^{r}=2 \rho\left(1-\rho^{2}\right)^{-2}$.

Theorem 4.3 clarifies the role of $\rho$ in shaping the correlation structure of a $\operatorname{SAR}(1)$ model. Since the contribution of a SAR-walk $\omega$ depends on $\rho$ only through $\rho^{\operatorname{len}(\omega)}$, it follows that: (i) when $|\rho|$ is "small", the covariance structure of a $\operatorname{SAR}(1)$ model is mostly determined by short SAR-walks; (ii) as $|\rho|$ increases, longer walks yield increasingly more important contributions, relative to shorter walks. In the rest of the paper we shall encounter several consequences of the fact that $\rho$ controls the relative importance of SAR-walks of different lengths.

More generally, Theorem 4.3 explains how correlations are formed in SAR(1) processes. Such an explanation contrasts with the conclusions in Wall (2004) that "there is no systematic structure to the SAR" model (p. 318) and that the correlation structure implied by the model "does not seem to follow an intuitive or practical scheme" (p. 321). In fact, the properties that are deemed to be counterintuitive in Wall (2004) can be understood using Theorem 4.3. For example, referring to a SAR(1) model on the graph of the United States and with a row-standardized $\boldsymbol{W}$, Wall states that "there does not appear to be any reason in general why a researcher would want to fit a spatial model that insists on Missouri and Tennessee 
being the least spatially correlated states in the land". Observe that row-standardization of $\boldsymbol{W}$ implies weights of arcs $(i, j)$ that are inversely proportional to the number of neighbors of $i$. Since both Missouri and Tennessee have several neighbors and are surrounded by states with several neighbors, it follows that any SAR-walk joining Missouri and Tennessee must have small weight. Thus, in any application where row-standardization is considered to be appropriate, Missouri and Tennessee must have low correlation, compared to other states.

In Wall (2004) the fact that a correlation implied by a SAR(1) model between two fixed units does not need to have the same sign for all $\rho \in\left(-\rho_{\max }, 0\right)$ is also regarded as counterintuitive. Again, Theorem 4.3 explains the reason behind such a behavior of the correlations. For any $\rho \in\left(-\rho_{\max }, 0\right)$ the SAR-walks of even length between $i$ and $j$ yield a positive contribution to $\operatorname{cov}\left(y_{i}, y_{j}\right)$, whereas SAR-walks of odd length yield a negative contribution. It follows that whether $\operatorname{corr}\left(y_{i}, y_{j}\right)$ is positive or negative for a particular value of $\rho$ depends on whether the total contribution of the SAR-walks of even length between $i$ and $j$ is larger or smaller than (minus) the total contribution of the SAR-walks of odd length. Which of the two total contributions is larger may depend on $\rho$, because, as we have seen above, the relative importance of SAR-walks of different lengths depends on $\rho .^{4}$

Even though our perspective is different from that of Wall (2004), we do agree with one of the remarks made in that paper, namely that it is impossible to fully understand the correlation structure of a SAR(1) model just by looking at $\boldsymbol{W} .{ }^{5}$ This point will be discussed in detail in the next section.

\section{The Correlations}

\subsection{An Expansion of the Correlations}

Starting from expression (5), it is straightforward to derive an expansion of $\operatorname{corr}\left(y_{i}, y_{j}\right)$ in powers of $\rho$.

Theorem 5.1 In a $S A R(1)$ model, when $|\rho| \lambda_{\max }<1$, and for any $i, j=1, \ldots, n$,

$$
\operatorname{corr}\left(y_{i}, y_{j}\right)=\sum_{r=0}^{\infty}\left[\left(\boldsymbol{Z}_{r}\right)_{i j}-b_{r}(i, j)\right] \rho^{r}
$$

with

$$
b_{r}(i, j):=\sum_{s=2}^{r}\left\{\left(\boldsymbol{Z}_{r-s}\right)_{i j} \sum_{\left(k_{2}, \ldots, k_{s}\right) \in \Phi}\left[\frac{\alpha_{K}}{k_{2} ! \ldots k_{s} !} \prod_{t=2}^{s}\left(\sum_{u=0}^{t}\left(\boldsymbol{Z}_{t-u}\right)_{i i}\left(\boldsymbol{Z}_{u}\right)_{j j}\right)^{k_{t}}\right]\right\},
$$

where $\Phi$ is the set of nonnegative $(s-1)$-tuples of integers $\left(k_{2}, \ldots, k_{s}\right)$ such that $\sum_{t=2}^{s} t k_{t}=s$, $K:=\sum_{t=2}^{s} k_{t}$, and $\alpha_{K}:=\prod_{l=0}^{K}(1 / 2-l)$.

Theorem 5.1 provides an exact expression for the correlations, in terms of $\rho$ and the entries of $\boldsymbol{W}$. The two terms that lead the expansion as $\rho \rightarrow 0$ are very simple. Indeed, by application of Lemma $4.2, b_{r}(i, j)=0$ if $r \leq d(i, j)+1$, and hence one obtains

$$
\operatorname{corr}\left(y_{i}, y_{j}\right)=\left(\boldsymbol{Z}_{d(i, j)}\right)_{i j} \rho^{d(i, j)}+\left(\boldsymbol{Z}_{d(i, j)+1}\right)_{i j} \rho^{d(i, j)+1}+O\left(\rho^{d(i, j)+2}\right),
$$


where, for two functions $f(x)$ and $g(x)$, we write $f(x)=O(g(x))$ to indicate that $|f(x) / g(x)|$ is bounded from above in some neighborhood of $x=0$.

Because, being a power series, (9) is uniformly convergent in a neighborhood of $\rho=0$, (11) specifies the low-order derivatives with respect to $\rho$ of the correlations at $\rho=0$. That is, we have $\left.\frac{\mathrm{d}^{r}}{\mathrm{~d} \rho^{r}} \operatorname{corr}\left(y_{i}, y_{j}\right)\right|_{\rho=0}=r !\left(\boldsymbol{Z}_{r}\right)_{i j}$, for any $r=1, \ldots, d(i, j)+1$. In particular,

$$
\left.\frac{\mathrm{d}}{\mathrm{d} \rho} \operatorname{corr}\left(y_{i}, y_{j}\right)\right|_{\rho=0}=\left(\boldsymbol{Z}_{1}\right)_{i j}=(\boldsymbol{W})_{i j}+(\boldsymbol{W})_{j i}
$$

which says that $\operatorname{corr}\left(y_{i}, y_{j}\right)$ has positive derivative at $\rho=0$ if $d(i, j)=1$ (i.e., if $i$ is a neighbor of $j$ or $j$ is a neighbor of $i$ ), zero derivative otherwise. According to expression (12) the magnitude of $(\boldsymbol{W})_{i j}+(\boldsymbol{W})_{j i}$ provides a ranking of all pairs $(i, j)$ such that $d(i, j)=1$ in terms of their degree of correlation as $\rho \rightarrow 0$. Note, however, that depending on $\boldsymbol{W}$ such a ranking does not need to hold for a fixed value of $\rho$. More specifically, setting $(\boldsymbol{W})_{i j}+$ $(\boldsymbol{W})_{j i}>(\boldsymbol{W})_{l m}+(\boldsymbol{W})_{m l}$ for some two pairs of units $(i, j)$ and $(l, m)$ does not necessarily imply that $\left|\operatorname{corr}\left(y_{i}, y_{j}\right)\right|>\left|\operatorname{corr}\left(y_{l}, y_{m}\right)\right|$ for some fixed value of $\rho$ (see, for example, Figures 2 and 3 below). What is more, the width of the interval of values of $\rho$ around 0 where $(\boldsymbol{W})_{i j}+(\boldsymbol{W})_{j i}>(\boldsymbol{W})_{l m}+(\boldsymbol{W})_{m l}$ does imply $\left|\operatorname{corr}\left(y_{i}, y_{j}\right)\right|>\left|\operatorname{corr}\left(y_{l}, y_{m}\right)\right|$ depends in a complicated way on $i, j, l, m$, and $\boldsymbol{W}$. The practical implication of such observations is that the specification of $\boldsymbol{W}$ does not allow the user of a SAR(1) model to impose that two variables $y_{i}$ and $y_{j}$ are more correlated than two other variables $y_{l}$ and $y_{m}$, because whether $\left|\operatorname{corr}\left(y_{i}, y_{j}\right)\right|$ is larger or smaller than $\left|\operatorname{corr}\left(y_{l}, y_{m}\right)\right|$ may depend on $\rho$, which is unknown and can only be estimated after $\boldsymbol{W}$ has been fixed.

\subsection{The Effect of Row-Standardization}

In economic applications it is common practice to standardize $\boldsymbol{W}$ so that all its row sums are 1 (assuming that $\boldsymbol{W}$ does not have any zero rows). As pointed out by Kelejian and Prucha (2010), some consequences of this practice are not completely understood. In this section we study the effect of row-standardization on the correlation structure of SAR(1) models. We focus on the correlations between units at distance 1, and on the case when, prior to row-standardization, the weights matrix is a $(0,1)$ matrix (that is, a matrix containing only zeros and ones) and is symmetric. Extensions of the results to other practically relevant cases can be obtained by a similar analysis, but are omitted for brevity.

We define $N_{i}:=\left\{j:(\boldsymbol{W})_{i j}>0\right\}, n_{i}:=\left|N_{i}\right|, n_{i j}:=\left|N_{i} \cap N_{j}\right|$, and $s_{i j}:=\sum_{l \in N_{i} \cap N_{j}} n_{l}^{-1}$.

Proposition 5.2 Consider a SAR(1) model with weights matrix $\boldsymbol{W}$, and let $i$ and $j$ be any two units such that $d(i, j)=1$.

(a) If $\boldsymbol{W}$ is a symmetric $(0,1)$ matrix, then

$$
\operatorname{corr}\left(y_{i}, y_{j}\right)=2 \rho+3 n_{i j} \rho^{2}+O\left(\rho^{3}\right)
$$

(b) if $\boldsymbol{W}$ is a row-standardized version of a symmetric $(0,1)$ matrix, then

$$
\operatorname{corr}\left(y_{i}, y_{j}\right)=\left(\frac{1}{n_{i}}+\frac{1}{n_{j}}\right) \rho+\left[\frac{n_{i j}}{n_{i} n_{j}}+\left(\frac{1}{n_{i}}+\frac{1}{n_{j}}\right) s_{i j}\right] \rho^{2}+O\left(\rho^{3}\right) .
$$


Despite their approximate nature, expressions (13) and (14) are helpful to clarify some consequences of row-standardizing $(0,1)$ weights matrices. Two such consequences are described next, and are then illustrated by means of a representative example.

Firstly, observe that the leading term in (13) is the same for each pair of neighbors, whereas that in (14) can be very different for different pairs of neighbors. Thus, up to order $O\left(\rho^{2}\right)$, different pairs of neighbors tend to be much more similarly correlated when $\boldsymbol{W}$ is a symmetric $(0,1)$ matrix than when it is row-standardized. A second consequence of rowstandardization relates to the approximate ranking of neighbors correlations implied by (13) and (14). Assuming that no edge corrections are implemented, the number of neighbors is generally larger for vertices in the central part of a graph (say between 4 and 6 for the rook's definition of adjacency in geographical applications) than for vertices close to the borders of the graph. Accordingly, the number $n_{i j}$ of common neighbors is generally larger for pairs of neighbors $(i, j)$ in the central part of a graph than for pairs of neighbors close to the borders of the graph, whereas the reverse holds for the quantity $n_{i}^{-1}+n_{j}^{-1}$. Thus, by expression (13), when $\boldsymbol{W}$ is a symmetric $(0,1)$ matrix and up to order $O\left(\rho^{3}\right)$, neighbors correlations are larger in the central part of the graph than at the borders. When $\boldsymbol{W}$ is row-standardized, the situation is reversed: according to (14), neighbors correlations are larger at the borders of the graph than in the central part, up to order $O\left(\rho^{2}\right)$. The intervals of values of $\rho$ around 0 where such implications hold depend on $\boldsymbol{W}$ in a complicated manner, but it is clear that the inequality $\left|\operatorname{corr}\left(y_{i}, y_{j}\right)\right| \geq\left|\operatorname{corr}\left(y_{l}, y_{m}\right)\right|$ is satisfied over a large interval if $n_{i j}-n_{l m}$ is large (for a $(0,1)$ weights matrix) or if $n_{i}^{-1}+n_{j}^{-1}-\left(n_{l}^{-1}+n_{m}^{-1}\right)$ is large (for a row-standardized weights matrix).

Example 5.3 Consider a random vector $\boldsymbol{y}$ observed over the map of the 48 continental United States, and suppose that two states are taken to be neighbors if and only if they share a common boundary or a common corner. Figure 2 displays all correlations implied by a $S A R(1)$ model between pairs of variables $y_{i}$ and $y_{j}$ such that states $i$ and $j$ are neighbors. The correlations are plotted for $\rho \in\left[0, \lambda_{\max }^{-1}\right)$, with $\lambda_{\max }^{-1}$ being about 0.185 for a $(0,1)$ weights matrix (panel (b)), 1 for the row-standardized case (panel (c)). Out of the 107 neighbor correlations, we have emphasized those between Missouri (MI) and Tennessee (TE), and between Maine (MA) and New Hampshire (NH). As predicted by our discussion above, when $\rho$ is small these two correlations are closer to each other when $\boldsymbol{W}$ is a $(0,1)$ matrix compared to the row-standardized case (e.g., for $\rho=.2 \lambda_{\max }^{-1}$ the correlations between $M I$ and TE and between $M A$ and $N H$ are respectively 0.08 and 0.07 if $\boldsymbol{W}$ is a $(0,1)$ matrix, whereas they are 0.06 and 0.26 after row-standardization). The number $n_{i j}$ of common neighbors is 2 for $M I$ and TE, 0 for MA and NH. Thus, by Proposition 5.2(a), MI and TE are more correlated than $M A$ and $N H$, when $\boldsymbol{W}$ is a $(0,1)$ matrix and for sufficiently small $\rho$. In fact, Figure 2(b) shows that this remains true for all $\rho \in\left(0, \lambda_{\max }^{-1}\right)$. A similar observation applies to the case of a row-standardized $\boldsymbol{W}$. The quantity $n_{i}^{-1}+n_{j}^{-1}$ equals $4 / 3$ for $M A$ and $N H$ and $1 / 4$ for MI and TE. Hence, by Proposition 5.2(b), MA and NH must be more correlated than Mi and Te for sufficiently small $\rho$; in fact, Figure 2(c) shows that this remains true unless $\rho$ is 
very close to 1.

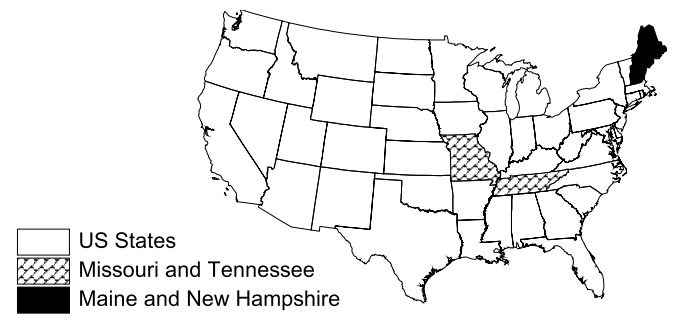

(a)

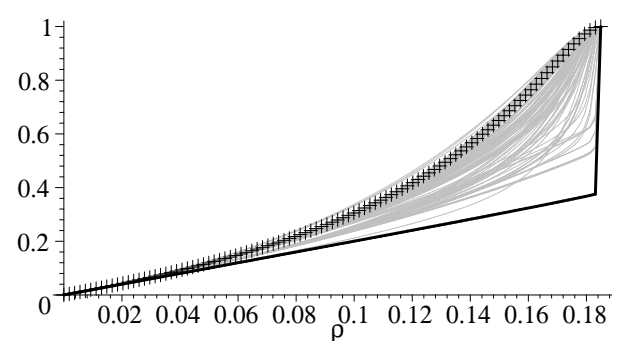

(b)

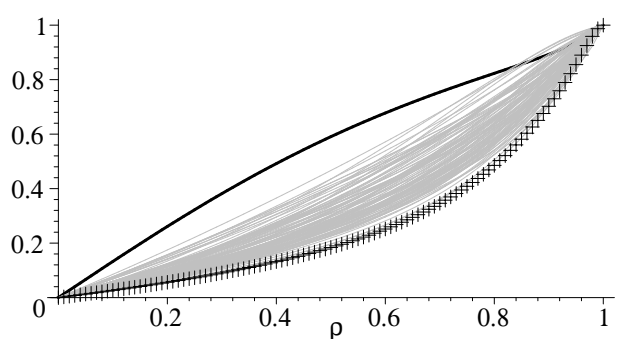

(c)

Figure 2: The correlations, as a function of $\rho$, implied by a $\operatorname{SAR}(1)$ model with $(0,1)$ weights matrix (panel (b)) and row-standardized weights matrix (panel (c)), for the 107 pairs of contiguous US, with emphasis on Missouri and Tennessee (crosses) and Maine and New Hampshire (dark solid line).

Following the usual rules for division of power series, from Proposition 5.2 it is possible to derive expansions for ratios of neighbors correlations. This may be useful to quickly compare the degrees of correlation of different pairs of neighbors. For instance, when $\boldsymbol{W}$ is a symmetric $(0,1)$ matrix expression (13) yields $\operatorname{corr}\left(y_{i}, y_{j}\right) / \operatorname{corr}\left(y_{l}, y_{m}\right)=1+\frac{3}{2}\left(n_{i j}-n_{l m}\right) \rho+O\left(\rho^{2}\right)$. In the case of Example 5.3, such an expression indicates that MI and TE $\left(n_{i j}=2\right)$ are $1+3 \rho$ times more correlated than MA and NH $\left(n_{l m}=0\right)$, up to order $O\left(\rho^{2}\right)$.

\subsection{Correlations as the Distance Changes}

We now turn to analyze how the correlations implied by a SAR(1) model depend on the graph distance $d(\cdot, \cdot)$. The following proposition establishes that, when $|\rho|$ is sufficiently small, the absolute value of $\operatorname{corr}\left(y_{i}, y_{j}\right)$ is inversely related to $d(i, j)$ for any $\boldsymbol{W}$.

Proposition 5.4 As $\rho \rightarrow 0$ in a $S A R(1)$ model, $\left|\operatorname{corr}\left(y_{i}, y_{j}\right)\right|>\left|\operatorname{corr}\left(y_{l}, y_{m}\right)\right|$ if $d(i, j)<$ $d(l, m)$, for any $i, j, l, m=1, \ldots, n$.

Similarly to other correlation properties discussed earlier, the ordering established by Proposition 5.4 does not need to hold over the whole parameter space of a SAR(1) model, and the interval where it holds depends on $\boldsymbol{W}$ and on $i, j, l, m$. An example is given next.

Example 5.5 Figure 3 displays $\operatorname{corr}\left(y_{i}, y_{j}\right)$ when $\boldsymbol{W}$ is a $(0,1)$ matrix and $i$ and $j$ are Maine and New Hampshire (darker line; $d(i, j)=1$ ) and Oklahoma and Nebraska (lighter line; $d(i, j)=2)$, for $0<\rho<\lambda_{\max }^{-1}$. For large values of $\rho$ the correlation between the units at distance 2 is much larger than the correlation between the units at distance 1. 


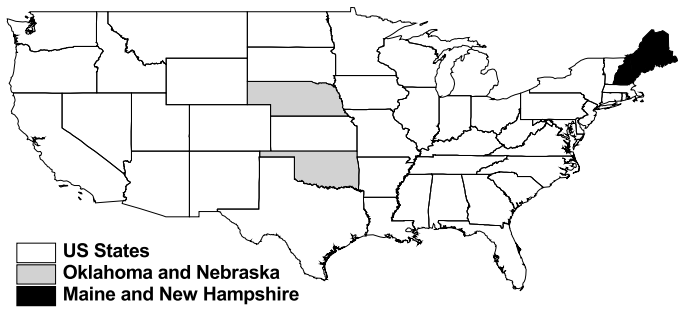

(a)

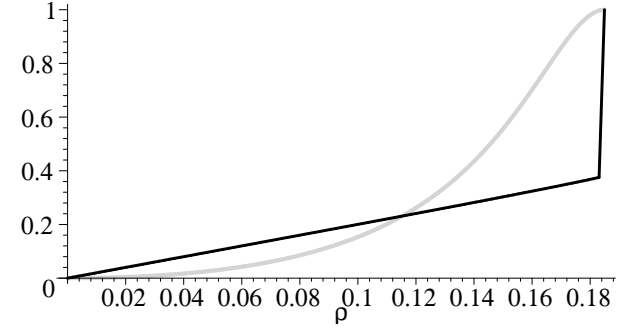

(b)

Figure 3: The correlations, as a function of $\rho$, implied by a $\operatorname{SAR}(1)$ model with $(0,1)$ weights matrix, for a pair of neighbors (darker line) and for a pair of non-neighbors (lighter line).

Figure 3 shows that, for large enough $\rho$, the correlations implied by a SAR(1) model are not guaranteed to be inversely related to the distance $d(\cdot, \cdot)$. In fact, it is generally impossible to impose that the correlations implied by a $\operatorname{SAR}(1)$ process are monotonically decreasing in some relevant distance. For the purpose of imposing correlation properties of this type, models that specify the covariance function directly in terms of some relevant distance (such as the so-called geostatistical models; see Cressie, 1993, Ch. 2) are clearly more suitable. It should be noted, however, that in many networks (e.g., social networks) (i) there is no reason why covariances should decrease monotonically in any distance, and (ii) no other relevant distance is available other than that induced by contiguity. In such cases, SAR(1) models may be more suitable than geostatistical models. For a comparison between SAR(1) models and geostatistical models, see, e.g., Cressie et al. (1999), Griffith and Csillag (1993), Song et al. (2008).

The possible nonmonotonicity of $\operatorname{SAR}(1)$ correlations in $d(\cdot, \cdot)$ originates from the fact that the contributions to a covariance given by long SAR-walks increase with $\rho$ (see Section 4). Recall however that the contribution of a SAR-walk depends not only on its length, but also on its weight. In particular, a specification of $\boldsymbol{W}$ such that SAR-walks have weights decaying quickly with their length may compensate for the effect of an increasing $\rho$. This is what happens with a row-standardized $\boldsymbol{W}$, because in that case the weight of a SAR-walk $\omega$ is inversely related to $n_{i}$, for each $i$ in $\omega$. Thus, one consequence of row-standardizing $\boldsymbol{W}$ is that it attenuates the possible nonmonotonicity of $\operatorname{corr}\left(y_{i}, y_{j}\right)$ in $d(i, j)$. Indeed, when the weights matrix of Example 5.5 is row-standardized, there is no value of $\rho$ such that a correlation between non-neighbors is much larger than a correlation between neighbors.

\subsection{Correlations at the Extremes of the Parameter Space}

This section analyzes the behavior of the correlations as $\rho$ approaches the extremes of the largest connected interval around the origin where $\boldsymbol{\Sigma}(\rho)$ exists. For this purpose, we assume $\lambda_{\max }>0$ and that $\boldsymbol{W}$ has at least one negative eigenvalue, so that the extremes are $\lambda_{\min }^{-1}$ and $\lambda_{\max }^{-1} \cdot{ }^{6}$

A natural question is whether two variables $y_{i}$ and $y_{j}$ approach perfect correlation as $\rho$ approaches $\lambda_{\min }^{-1}$ or $\lambda_{\max }^{-1}$. Let us denote by $g_{\min }$ (resp. $g_{\max }$ ) the geometric multiplicity of 
$\lambda_{\min }\left(\right.$ resp. $\lambda_{\max }$ ), and by $\boldsymbol{q}_{\min }$ (resp. $\boldsymbol{q}_{\max }$ ) an arbitrary eigenvector of $\boldsymbol{W}$ associated to $\lambda_{\min }$ (resp. $\left.\lambda_{\max }\right)$. In most applications $g_{\min }$ and $g_{\max }$ are $1 .^{7}$

Theorem 5.6 In a $S A R(1)$ model, for any $\boldsymbol{W}$ and for any $i, j=1, \ldots, n$,

(a) if $g_{\min }=1$ and $\left(\boldsymbol{q}_{\min }\right)_{i}\left(\boldsymbol{q}_{\min }\right)_{j}>0$, then $\lim _{\rho \rightarrow \lambda_{\min }^{-1}} \operatorname{corr}\left(y_{i}, y_{j}\right) \rightarrow 1$;

(b) if $g_{\min }=1$ and $\left(\boldsymbol{q}_{\min }\right)_{i}\left(\boldsymbol{q}_{\min }\right)_{j}<0$, then $\lim _{\rho \rightarrow \lambda_{\min }^{-1}} \operatorname{corr}\left(y_{i}, y_{j}\right) \rightarrow-1$;

(c) if $g_{\max }=1$ and $\left(\boldsymbol{q}_{\max }\right)_{i}\left(\boldsymbol{q}_{\max }\right)_{j} \neq 0$, then $\lim _{\rho \rightarrow \lambda_{\max }^{-1}} \operatorname{corr}\left(y_{i}, y_{j}\right) \rightarrow 1$.

In the cases not contemplated by Theorem 5.6 the limiting behavior of the correlations at $\lambda_{\min }^{-1}$ and $\lambda_{\max }^{-1}$ depends on $\boldsymbol{W}$. More specifically, if $\left(\boldsymbol{q}_{\min }\right)_{i}\left(\boldsymbol{q}_{\min }\right)_{j}=0$ or $g_{\min }>$ 1 , then $\operatorname{corr}\left(y_{i}, y_{j}\right)$ can approach any value in $[-1,1]$ as $\rho \rightarrow \lambda_{\min }^{-1}$, depending on $\boldsymbol{W}$; if $\left(\boldsymbol{q}_{\max }\right)_{i}\left(\boldsymbol{q}_{\max }\right)_{j}=0$ or $g_{\max }>1$, then $\operatorname{corr}\left(y_{i}, y_{j}\right)$ can approach any value in $[0,1]$ as $\rho \rightarrow \lambda_{\max }^{-1}$, depending on $\boldsymbol{W}$ (cf. Martellosio, 2011, Lemma 3.3).

In the rest of this section we focus on the right extreme $\lambda_{\max }^{-1}$, because positive autocorrelation is much more common in applications than negative autocorrelation, and because a SAR(1) model with $\rho$ close to $\lambda_{\max }^{-1}$ has an intrinsic interest due to the analogy with the near unit root case in an AR(1) model; see, e.g., Lee and Yu (2011). The following result gives a sufficient condition for all pairs of variables to approach perfect correlation as $\rho \rightarrow \lambda_{\max }^{-1}$. It should be noted that the condition is given in terms of walks, not SAR-walks.

Corollary 5.7 If there is a walk from each unit to every other unit, then all correlations implied by a $S A R(1)$ model tend to 1 as $\rho \rightarrow \lambda_{\max }^{-1}$.

It easy to see that the condition in Corollary 5.7 is satisfied if and only if there is no ordering of the units such that $\boldsymbol{W}$ is block-triangular. If such an ordering exists, which is often the case in applications to directed networks, then there may be correlations that do not approach 1 as $\rho \rightarrow \lambda_{\max }^{-1}$.

Example 5.8 Figure 4 displays corr $\left(y_{2}, y_{4}\right)$ implied by a SAR(1) model with the blocktriangular weights matrix of Example 3.2, for $0 \leq \rho<\lambda_{\max }^{-1}=1$. Observe that $\operatorname{corr}\left(y_{2}, y_{4}\right)$ does not approach 1 as $\rho \rightarrow 1$.

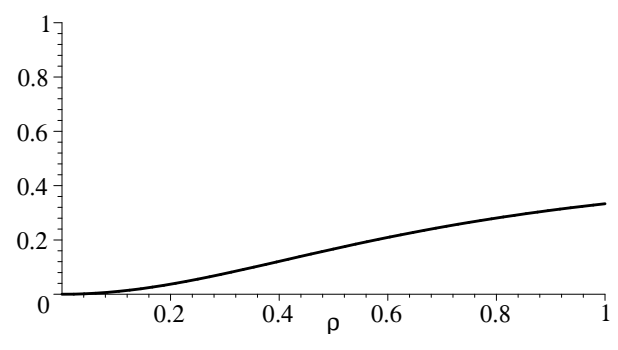

Figure 4: $\operatorname{corr}\left(y_{2}, y_{4}\right)$ implied by a $\operatorname{SAR}(1)$ model with weights matrix (3). 
In the case of an undirected graph a block-triangular $\boldsymbol{W}$ must be block-diagonal. Thus, by Corollary 5.7, all correlations implied by a SAR(1) model on an undirected graph tend to 1 as $\rho \rightarrow \lambda_{\max }^{-1}$ if there is no ordering of the units such that $\boldsymbol{W}$ is block-diagonal. Block-diagonal weights matrices are important in some econometric applications; see, e.g., Case (1992). ${ }^{8}$

Corollary 5.9 Let $\boldsymbol{W}$ be a block-diagonal weights matrix such that there is a walk from each unit to every other unit in the same block. If $\boldsymbol{W}$ is row-standardized, then all SAR(1) correlations relative to units within the same block tend to 1 as $\rho \rightarrow 1$.

We now point out an interesting connection between Theorem 5.6(c) (and its two corollaries) and a particular concept of centrality in a network. Such a connection rests on the fact that $\left(\boldsymbol{q}_{\max }\right)_{i}$ is a relative measure of the involvement of $i$ in the the walk structure of the graph underlying $\boldsymbol{W}$, known as the eigenvector centrality of vertex $i$; see, e.g., Bonacich (1972). If $\left(\boldsymbol{q}_{\max }\right)_{i}$ and $\left(\boldsymbol{q}_{\max }\right)_{j}$ are nonzero but very close to zero (compared to all other entries of $\left.\boldsymbol{q}_{\max }\right)$, then corr $\left(y_{i}, y_{j}\right)$ approaches 1 slowly and therefore Theorem 5.6(c) may be uninformative about the behavior of $\operatorname{corr}\left(y_{i}, y_{j}\right)$ in a neighborhood of $\lambda_{\max }^{-1}$. Since a small $\left(\boldsymbol{q}_{\text {max }}\right)_{i}$ indicates low centrality, this means that $\operatorname{corr}\left(y_{i}, y_{j}\right)$ approaches 1 slowly as $\rho \rightarrow \lambda_{\max }^{-1}$ if $i$ and $j$ have low centrality. For example, the correlation between Maine and New Hampshire in Figure 2(b) does approach 1 as $\rho \rightarrow \lambda_{\max }^{-1}$, but remains less than 0.4 unless $\rho$ is very close to $\lambda_{\max }^{-1} \cdot{ }^{9}$ Note that such nonsmoothness of $\operatorname{corr}\left(y_{i}, y_{j}\right)$ for $\rho$ close to $\lambda_{\max }^{-1}$ does not occur when $\boldsymbol{W}$ is row-standardized, because in that case $\boldsymbol{q}_{\max }$ is a vector of identical entries, and hence all units have the same centrality.

\section{Conclusion}

Especially when defined on an irregular lattice, a SAR(1) process generates a complicated correlation structure that can be interpreted as the equilibrium of an influence process between all spatial units. We have shown that the correlations implied by a SAR(1) model can be understood in terms of a particular type of walks between the observational units, which we have named SAR-walks. Each SAR-walk between two units gives a contribution to the covariance between the random variables observed at those units, with the parameter $\rho$ controlling the relative importance of the contributions coming from SAR-walks of different lengths.

A natural extension of our analysis is to study how the correlation properties of a $\operatorname{SAR}(1)$ process are affected by the presence of group theoretic symmetries and combinatorial regularities in the underlying graph. We will report our work on this theme elsewhere.

Finally, it is worth mentioning that all the results given in this paper can be readily extended to first-order conditional autoregressive (CAR(1)) processes (e.g., Besag, 1974; Cressie, 1993). CAR(1) processes share some similarities with SAR(1) processes, and are very popular in many fields, for instance disease mapping and image analysis, but not in economics. In fact, the analysis of CAR(1) processes is simpler, because in order to study their correlation structure the weights matrix can be taken to be symmetric without loss of 
generality, and hence walks and their weights can be defined without regards to the direction of their steps.

\section{Notes}

${ }^{1}$ There are several such extensions, e.g., the spatial lag, spatial error, or spatial Durbin models (see, for instance, Anselin, 1988). All these models have variance matrix (2). For an interpretation of the marginal effects in a spatial Durbin model that has some similarities with the interpretation of the correlation structure developed in this paper, see LeSage and Pace (2009), Section 2.7.

${ }^{2}$ We can take $\boldsymbol{V}=\boldsymbol{I}_{n}$ because the correlation matrix of $\boldsymbol{y}$ is invariant to transformations $\boldsymbol{y} \rightarrow \boldsymbol{T} \boldsymbol{y}$, for any diagonal matrix $\boldsymbol{T}$ with positive diagonal entries (if $\boldsymbol{V} \neq \boldsymbol{I}_{n}$, just take $\boldsymbol{T}=\boldsymbol{V}^{-1 / 2}$ ). The results in this paper continue to hold when $\boldsymbol{V} \neq \boldsymbol{I}_{n}$, with $\boldsymbol{W}$ replaced with $\boldsymbol{V}^{-1 / 2} \boldsymbol{W} \boldsymbol{V}^{1 / 2}$.

${ }^{3}$ The case $\rho_{\min }=-\infty$ is relevant when $\boldsymbol{W}$ is nilpotent, or when each eigenvalue of $\boldsymbol{W}$ is either nonnegative or complex. On the other hand, $\lambda_{\max }=0$ occurs if and only if $\boldsymbol{W}$ is nilpotent. Thus, when $\boldsymbol{W}$ is non-nilpotent, $\lambda_{\max }$ can be taken to be equal to 1 without loss of generality, by a reparametrization of the model.

${ }^{4}$ It is worth noting that there always exists a left neighborhood of $\rho=0$ where $\operatorname{corr}\left(y_{i}, y_{j}\right)>0$ if $d(i, j)$ is even, $\operatorname{corr}\left(y_{i}, y_{j}\right)<0$ if $d(i, j)$ is odd. This is because the lowest power of $\rho$ in $(6)$ is $d(i, j)$, by the definition of $d(\cdot, \cdot)$.

${ }^{5}$ There are of course some correlation properties that can be inferred from $\boldsymbol{W}$ alone (e.g., for any $\rho \in$ $\left(0, \rho_{\max }\right)$, all correlations are nonnegative, and they are positive if the graph underlying $\boldsymbol{W}$ is strongly connected), but these are all rather weak properties.

${ }^{6}$ When $\lambda_{\max }=0$, the right extreme of the parameter space is $\infty$ (see Section 2). Similarly, when $\boldsymbol{W}$ does not have any negative eigenvalues, the left extreme is $-\infty$. We do not consider these two cases because the behavior of the correlations as $\rho \rightarrow \pm \infty$ is not very interesting from a practical point of view.

${ }^{7}$ More precisely, $g_{\min }>1$ or $g_{\max }>1$ generally require $\boldsymbol{W}$ to satisfy some symmetries (e.g., Biggs, 1993, Ch. 15), and $g_{\max }>1$ also requires reducibility of $\boldsymbol{W}$. One example of a weights matrix that satisfies several symmetries and is sometimes used in social network analysis is a block diagonal matrix whose main diagonal blocks have off-diagonal entries equal to 1 . For such a matrix, $g_{\min }=n-r$ and $g_{\max }=r$, where $r$ is the number of main diagonal blocks.

${ }^{8}$ It is worth pointing out that in some cases it may be appropriate to use different autoregressive parameters for different blocks.

${ }^{9}$ When $\boldsymbol{q}_{\text {max }}$ is normalized to have length 1 , the entry of $\boldsymbol{q}_{\max }$ corresponding to Maine is $1.39 \cdot 10^{-7}$ and that corresponding to New Hampshire is $4.08 \cdot 10^{-6}$.

\section{Appendix A Proofs}

We first give an auxiliary lemma, and then prove all results stated in the main text.

Lemma A.1 Let $\lambda$ be an eigenvalue of an $n \times n$ matrix $\boldsymbol{M}$, with geometric multiplicity $g_{\lambda}$. Then, $\operatorname{rank}\left(\left(\boldsymbol{I}_{n}-\lambda^{-1} \boldsymbol{M}^{\prime}\right)\left(\boldsymbol{I}_{n}-\lambda^{-1} \boldsymbol{M}\right)\right)=n-g_{\lambda}$.

Proof. For any matrix $\boldsymbol{B}, \operatorname{rank}\left(\boldsymbol{B}^{\prime} \boldsymbol{B}\right)=\operatorname{rank}(\boldsymbol{B})$ and $\operatorname{rank}(\boldsymbol{B})+\operatorname{dim}(\mathcal{N}(\boldsymbol{B}))=n$, where $\mathcal{N}(\boldsymbol{B})$ denotes the nullspace of $\boldsymbol{B}$. The result follows, because $g_{\lambda}:=\operatorname{dim}\left(\mathcal{N}\left(\boldsymbol{M}-\lambda \boldsymbol{I}_{n}\right)\right)$.

Proof of Lemma 4.1. The lemma is trivial for $r=0$ or for $r=1$, so let us assume $r>1$. On expanding the products of the $k$ matrices $\boldsymbol{W}$ and $r-k$ matrices $\boldsymbol{W}^{\prime}$ that appears in expression (4), the $i j$-th entry of $\boldsymbol{Z}_{r}$ can be expressed as

$$
\left(\boldsymbol{Z}_{r}\right)_{i j}=\sum_{k=0}^{r} \sum_{l_{1}, \ldots, l_{r-1}=1}^{n} a\left(k, i, l_{1}, \ldots, l_{r-1}, j\right),
$$


where, taking for notational convenience $k \in(2, r-2)$,

$$
\begin{aligned}
& a\left(k, i, l_{1}, \ldots, l_{r-1}, j\right)=(\boldsymbol{W})_{i l_{1}}(\boldsymbol{W})_{l_{1} l_{2} \ldots}(\boldsymbol{W})_{l_{k-1} l_{k}}\left(\boldsymbol{W}^{\prime}\right)_{l_{k} l_{k+1} \ldots}\left(\boldsymbol{W}^{\prime}\right)_{l_{r-2} l_{r-1}}\left(\boldsymbol{W}^{\prime}\right)_{l_{r-1} j}
\end{aligned}
$$

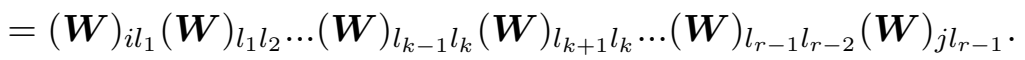

The modification of (16) required for $0 \leq k \leq 2$ or $r-2 \leq k \leq r$ is obvious. Observe now that, for any $k=0, \ldots, r, a\left(k, i, l_{1}, \ldots, l_{r-1}, j\right)$ is equal to the weight of the SAR-walk $\left(i \rightarrow l_{1} \rightarrow \ldots \rightarrow l_{k} \leftarrow \ldots \leftarrow l_{r-1} \leftarrow j\right)$ if such a SAR-walk exists, to 0 otherwise. Thus, by (15), $\left(\boldsymbol{Z}_{r}\right)_{i j}$ is equal to the sum of the weights of all SAR-walks of length $r$ from $i$ to $j$.

Proof of Lemma 4.2. Follows from the combination of Definition 3.3 and Lemma 4.1.

Proof of Theorem 4.3. Let $\mathcal{K}_{i j}^{(r)}$ denote the set of all SAR-walks of length $r$ from $i$ to $j$, so that, according to Lemma 4.1, $\left(\boldsymbol{Z}_{r}\right)_{i j}=\sum_{\omega \in \mathcal{K}_{i j}^{(r)}}$ wei $(\omega)$. Using expression (5), we obtain that, when $|\rho| \lambda_{\max }<1, \operatorname{cov}\left(y_{i}, y_{j}\right)=\sum_{r=d(i, j)}^{\infty} \sum_{\omega \in \mathcal{K}_{i j}^{(r)}} \operatorname{wei}(\omega) \rho^{r}=\sum_{\omega \in \mathcal{K}_{i j}} \operatorname{wei}(\omega) \rho^{\operatorname{len}(\omega)}$.

Proof of Theorem 5.1. Let $\boldsymbol{\Sigma}_{i j}^{*}(\rho)$ denote $\operatorname{corr}\left(y_{i}, y_{j}\right)$ in a $\operatorname{SAR}(1)$ model, $D^{r} f(x)$ denote the $r$-th derivative of a function $f: \mathbb{R} \rightarrow \mathbb{R}$, and $D^{r} f(0)$ denote $D^{r} f(x)$ evaluated at $x=0$. In any neighborhood of $\rho=0$ such that $|\rho| \lambda_{\max }<1, \boldsymbol{\Sigma}_{i j}^{*}(\rho)$ is an infinitely differentiable function of $\rho$, and hence it admits the MacLaurin expansion $\sum_{r=0}^{\infty}(r !)^{-1} D^{r} \boldsymbol{\Sigma}_{i j}^{*}(0) \rho^{r}$. The bulk of the proof consists of expressing the coefficients $(r !)^{-1} D^{r} \boldsymbol{\Sigma}_{i j}^{*}(0)$ in terms of entries of the matrices $\boldsymbol{Z}_{0}, \ldots, \boldsymbol{Z}_{r}$. Write $\boldsymbol{\Sigma}_{i j}^{*}(\rho)=\boldsymbol{\Sigma}_{i j}(\rho) \eta(v(\rho))$, with $\eta(z):=z^{-1 / 2}$ and $v(\rho):=\boldsymbol{\Sigma}_{i i}(\rho) \boldsymbol{\Sigma}_{j j}(\rho)$. By Leibniz's formula,

$$
\frac{1}{r !} D^{r} \boldsymbol{\Sigma}_{i j}^{*}(0)=\sum_{s=0}^{r} \frac{1}{s !(r-s) !} D^{r-s} \boldsymbol{\Sigma}_{i j}(0) D^{s} \eta(v(0)),
$$

where, applying Faá di Bruno's formula (e.g., Abramowitz and Stegun, 1979),

$$
D^{s} \eta(v(0))=\sum_{\left(k_{2}, \ldots, k_{s}\right) \in \Phi} \frac{s !}{k_{1} ! \ldots k_{s} !}\left(D^{k} \eta\right)(v(0)) \prod_{t=1}^{s}\left(\frac{D^{t} v(0)}{t !}\right)^{k_{t}}
$$

with $k:=\sum_{t=1}^{s} k_{t}$. Since $v(0)=1$ and $D^{k} \eta(z)=\prod_{l=0}^{k-1}(1 / 2-l) z^{-1 / 2-k}$, it follows that $\left(D^{k} \eta\right)(v(0))=\prod_{l=0}^{k-1}(1 / 2-l)$. In addition, from expression (5), we have that, for $|\rho| \lambda_{\max }<$ 1, $v(\rho)=\sum_{r, s=0}^{\infty} \rho^{r+s}\left(\boldsymbol{Z}_{r}\right)_{i i}\left(\boldsymbol{Z}_{s}\right)_{j j}=\sum_{r=0}^{\infty} \rho^{r} \sum_{u=0}^{r}\left(\boldsymbol{Z}_{r-u}\right)_{i i}\left(\boldsymbol{Z}_{u}\right)_{j j}$, and hence $D^{t} v(0) / t !=$ $\sum_{u=0}^{t}\left(\boldsymbol{Z}_{t-u}\right)_{i i}\left(\boldsymbol{Z}_{u}\right)_{j j}$. Thus, since $D^{1} v(0)=0$, (18) yields $D^{s} \eta(v(0))=0$ if $s=1$, and

$$
D^{s} \eta(v(0))=\sum_{\left(k_{2}, \ldots, k_{s}\right) \in \Phi} \frac{s ! \alpha_{K}}{k_{2} ! \ldots k_{s} !} \prod_{t=2}^{s}\left(\sum_{u=0}^{t}\left(\boldsymbol{Z}_{t-u}\right)_{i i}\left(\boldsymbol{Z}_{u}\right)_{j j}\right)^{k_{t}}
$$

if $s>1$. Substituting expressions (19) and $D^{r-s} \boldsymbol{\Sigma}_{i j}(0)=(r-s) !\left(\boldsymbol{Z}_{r-s}\right)_{i j}$ into (17) gives $(r !)^{-1} D^{r} \boldsymbol{\Sigma}_{i j}^{*}(0)=\left(\boldsymbol{Z}_{r}\right)_{i j}-b_{r}(i, j)$, which is the desired result.

Proof of Proposition 5.2. Both parts (a) and (b) are obtained by substituting the relevant expressions for $\left(\boldsymbol{Z}_{1}\right)_{i j}$ and $\left(\boldsymbol{Z}_{2}\right)_{i j}$ into (11). If $\boldsymbol{W}$ is a symmetric $(0,1)$ matrix, then, for any pair of neighbors $(i, j),\left(\boldsymbol{Z}_{1}\right)_{i j}=2$ and $\left(\boldsymbol{Z}_{2}\right)_{i j}=3\left(\boldsymbol{W}^{2}\right)_{i j}=3 \sum_{l=1}^{n} \boldsymbol{W}_{i l} \boldsymbol{W}_{l j}=3 n_{i j}$. 
Consider now a row-standardized weights matrix $\boldsymbol{W}=\boldsymbol{D}^{-1} \boldsymbol{A}$, where $\boldsymbol{A}$ is a symmetric $(0,1)$ matrix, and $\boldsymbol{D}$ is the diagonal matrix with $(\boldsymbol{D})_{i i}=\sum_{j=1}^{n}(\boldsymbol{A})_{i j}, i=1, \ldots, n$. For any pair of neighbors $(i, j),\left(\boldsymbol{Z}_{1}\right)_{i j}=n_{i}^{-1}(\boldsymbol{A})_{i j}+n_{j}^{-1}(\boldsymbol{A})_{j i}=n_{i}^{-1}+n_{j}^{-1}$ and $\left(\boldsymbol{Z}_{2}\right)_{i j}=$ $\left(\boldsymbol{W}^{2}\right)_{j i}+\left(\boldsymbol{W} \boldsymbol{W}^{\prime}\right)_{i j}+\left(\boldsymbol{W}^{2}\right)_{i j}$, where $\left(\boldsymbol{W} \boldsymbol{W}^{\prime}\right)_{i j}=\left(\boldsymbol{D}^{-1} \boldsymbol{A} \boldsymbol{A} \boldsymbol{D}^{-1}\right)_{i j}=\left(n_{i} n_{j}\right)^{-1} \sum_{l=1}^{n} \boldsymbol{A}_{i l} \boldsymbol{A}_{l j}=$ $\left(n_{i} n_{j}\right)^{-1} n_{i j},\left(\boldsymbol{W}^{2}\right)_{j i}=n_{j}^{-1} s_{i j}$, and $\left(\boldsymbol{W}^{2}\right)_{i j}=\left(\boldsymbol{D}^{-1} \boldsymbol{A} \boldsymbol{D}^{-1} \boldsymbol{A}\right)_{i j}=n_{i}^{-1} \sum_{l=1}^{n} n_{l}^{-1}(\boldsymbol{A})_{i l}(\boldsymbol{A})_{l j}=$ $n_{i}^{-1} s_{i j}$.

Proof of Proposition 5.4. By Lemma 4.2 and Assumption 2.1, $\left(\boldsymbol{Z}_{d(i, j)}\right)_{i j}>0$. Hence, Theorem 5.1 implies that the larger $d(i, j)$ is, the faster $\operatorname{corr}\left(y_{i}, y_{j}\right)$ goes to zero as $\rho \rightarrow 0$.

Proof of Theorem 5.6. For any $\rho \in\left(\lambda_{\min }^{-1}, \lambda_{\max }^{-1}\right)$, let $\gamma_{1}(\rho)<\gamma_{2}(\rho)<\ldots<\gamma_{s}(\rho)$ denote the distinct eigenvalues of $\boldsymbol{\Sigma}^{-1}(\rho)$. Note that $s$ does not depend on $\rho$ except for a finite number of values of $\rho$ (see Kato, 1995, p. 64). By Lemma A.1 in Martellosio (2011), the eigenspace of $\boldsymbol{\Sigma}^{-1}\left(\lambda_{\max }^{-1}\right)$ associated to $\gamma_{1}\left(\lambda_{\max }^{-1}\right)=0$ is equal to the eigenspace, say $\boldsymbol{E}_{\lambda_{\max }}$, of $\boldsymbol{W}$ associated to $\lambda_{\max }$. Assume now that $g_{\max }=1$. Then the eigenspace of $\boldsymbol{\Sigma}^{-1}\left(\lambda_{\max }^{-1}\right)$ associated to $\gamma_{1}\left(\lambda_{\max }^{-1}\right)$ is 1-dimensional. This, together with continuity of the functions $\gamma_{l}(\rho)$, implies that Assumption 2 in Martellosio (2011) is satisfied. Thus, by Lemma 3.3 in Martellosio (2011), $\gamma_{1}(\rho) \boldsymbol{\Sigma}(\rho) \rightarrow \boldsymbol{E}_{\lambda_{\max }}$ as $\rho \rightarrow \lambda_{\max }^{-1}$. Since $\boldsymbol{E}_{\lambda_{\max }}=\left(\boldsymbol{q}_{\max } \boldsymbol{q}_{\max }^{\prime}\right) /\left(\boldsymbol{q}_{\max }^{\prime} \boldsymbol{q}_{\max }\right)$, it follows that $\gamma_{1}(\rho) \operatorname{cov}\left(y_{i}, y_{j}\right) \rightarrow\left(\boldsymbol{q}_{\text {max }}\right)_{i}\left(\boldsymbol{q}_{\text {max }}^{\prime}\right)_{j} /\left(\boldsymbol{q}_{\text {max }}^{\prime} \boldsymbol{q}_{\text {max }}\right)$. Hence, provided that $\left(\boldsymbol{q}_{\text {max }}\right)_{i}\left(\boldsymbol{q}_{\text {max }}\right)_{j} \neq 0$, and using $\operatorname{corr}\left(y_{i}, y_{j}\right)=\operatorname{cov}\left(y_{i}, y_{j}\right) /\left(\operatorname{var}\left(y_{i}\right) \operatorname{var}\left(y_{j}\right)\right)^{1 / 2}$, one obtains

$$
\lim _{\rho \rightarrow \lambda_{\max }^{-1}} \operatorname{corr}\left(y_{i}, y_{j}\right) \rightarrow\left(\boldsymbol{q}_{\max }\right)_{i}\left(\boldsymbol{q}_{\max }\right)_{j} /\left|\left(\boldsymbol{q}_{\max }\right)_{i}\left(\boldsymbol{q}_{\max }\right)_{j}\right|=\operatorname{sgn}\left(\left(\boldsymbol{q}_{\max }\right)_{i}\left(\boldsymbol{q}_{\max }\right)_{j}\right) .
$$

Part (c) of the theorem follows, because, by Assumption 2.1 and Theorem 8.3.1 of Horn and Johnson (1985), $g_{\max }=1$ implies that $\boldsymbol{q}_{\max }$ is entrywise nonnegative or nonpositive. By a straightforward extension of the arguments above equation (20), one can easily see that, if $g_{\min }=1$ and $\left(\boldsymbol{q}_{\min }\right)_{i}\left(\boldsymbol{q}_{\min }\right)_{j} \neq 0$,

$$
\lim _{\rho \rightarrow \lambda_{\min }^{-1}} \operatorname{corr}\left(y_{i}, y_{j}\right) \rightarrow \operatorname{sgn}\left(\left(\boldsymbol{q}_{\min }\right)_{i}\left(\boldsymbol{q}_{\min }\right)_{j}\right),
$$

which proves parts (a) and (b) of the theorem.

Proof of Corollary 5.7. If there is a walk from each unit to every other unit, then $\boldsymbol{W}$ is an irreducible matrix. Since $\boldsymbol{W}$ is nonnegative, the Perron-Frobenius Theorem implies that $g_{\text {max }}=1$ and $\boldsymbol{q}_{\max }$ is entrywise positive (see, Horn and Johnson, 1985). The desired result follows from Theorem 5.6(c).

Proof of Corollary 5.9. A SAR(1) model with block-diagonal weights matrix can be decomposed into the product of a number of submodels equal to the number of diagonal blocks. Since there is a walk from each unit to every other unit in the same block, $\boldsymbol{W}$ does not have any zero rows, and can therefore be row-standardized, which implies $\lambda_{\max }=1$. By Corollary 5.7, all correlations implied by each submodel tend to 1 as $\rho \rightarrow 1$.

\section{References}

Abramowitz, A. \& S. Stegun (1979) Handbook of Mathematical Functions. Nauka. 
Anselin, L. (1988) Spatial Econometrics: Methods and Models. Kluwer.

Bartlett, M. (1975) The Statistical Analysis of Spatial Pattern. Chapman-Hall.

Bavaud, F. (1998) Models for Spatial Weights: A Systematic Look. Geographical Analysis 50, 155-71.

Besag, J.E. (1972) On the correlation structure of some two-dimensional stationary processes. Biometrika 59, 43-48.

Besag, J.E. (1974) Spatial interaction and the statistical analysis of lattice data. Journal of the Royal Statistical Society B 36, 192-236.

Biggs, N.L. (1993) Algebraic Graph Theory, second edition. Cambridge University Press.

Bonacich, P. (1972) Factoring and weighting approaches to status scores and clique identification. Journal of Mathematical Sociology 2, 113-120.

Case, A. (1992) Neighborhood influence and technological change. Regional Science and Urban Economics 22, 491-508.

Cliff, A.D. \& J.K. Ord (1981) Spatial Processes: Models and Applications. Pion.

Cressie, N. (1993) Statistics for Spatial Data. Wiley, New York.

Cressie, N., M.S. Kaiser, M.J. Daniels, J. Aldworth, J. Lee, S.N. Lahiri, \& L.H. Cox (1999) Spatial analysis of particulate matter in an urban environment. In J. Gomez-Hernandez, A. Soares, and R. Froidevaux (eds), geoENV II - Geostatistics for Environmental Applications, pp. 41-52. Kluwer.

Godsil, C. (1993) Algebraic Combinatorics. Chapman and Hall.

Griffith, D.A. \& F. Csillag (1993) Exploring relationships between semivariogram and spatial autoregressive models. Papers in Regional Science 72, 283-295.

Griffith, D.A. \& F. Lagona (1998) On the quality of likelihood based estimators in spatial autoregressive models when the data dependence structure is misspecified. Journal of Statistical Planning and Inference 69, 153-174.

Harary, F.H. (1969) Graph Theory. Addison-Wesley.

Horn, R. \& C.R. Johnson (1985) Matrix Analysis. Cambridge University Press.

Kato, T. (1995) Perturbation Theory for Linear Operators. Springer.

Kelejian, H.H. \& I.R. Prucha (2010) Specification and estimation of spatial autoregressive models with autoregressive and heteroskedastic disturbances. Journal of Econometrics 157, 53-67.

Lee, L.F. \& J. Yu (2011) Near unit root in the spatial autoregressive model. Manuscript, The Ohio State University.

LeSage, J. \& R.K. Pace (2009) Introduction to Spatial Econometrics. Taylor and Francis/CRC.

Martellosio, F. (2011) Efficiency of the OLS estimator in the vicinity of a spatial unit root. Statistics \& Probability Letters 81, 1285-1291.

Song, H.R., M. Fuentes \& S. Ghosh (2008) A comparative study of Gaussian geostatistical models and Gaussian Markov random field models. Journal of Multivariate Analysis 99, 1681-1697. 
Wall, M.M. (2004) A close look at the spatial structure implied by the CAR and SAR models. Journal of Statistical Planning and Inference 121, 311-324.

Whittle, P. (1954) On stationary processes in the plane. Biometrika 41, 434-449. 